\title{
Systemic Lupus Erythematosus Nephritis Class IV S
}

National Cancer Institute

\section{Source}

National Cancer Institute. Systemic Lupus Erythematosus Nephritis Class IV S. NCI

Thesaurus. Code C123120.

Systemic lupus erythematosus nephritis characterized by active or inactive diffuse, segmental endo- or extracapillary glomerulonephritis that involves 50\% or more of all glomeruli. (Weening, Jan J. et al. (2004). The Classification of Glomerulonephritis in Systemic Lupus Erythematosus Revisited. Journal of the American Society of Nephrology 15(2), 241-50.) 